\title{
Reservoir fluid substitution effects on seismic profile interpretation: a physical modeling experiment
}

\author{
Shangxu Wang ${ }^{1}$, Xiang-Yang $\mathrm{Li}^{2}$, Bangrang $\mathrm{Di}^{1}$, David Booth ${ }^{2}$ \\ ${ }^{1}$ National Key Laboratory of Petroleum Resources and Prospecting, China University of \\ Petroleum \\ ${ }^{2}$ British Geological Survey
}

P-wave seismic surveys are performed on realistic physical models of a reservoir formed of interbedded sand and shale layers and filled with gas, water, and oil. They show characteristic behaviour which can be used to distinguish between fluids, with significant implications for the interpretation of time-lapse experiments. Discontinuous reflections originate in gas-filled reservoirs, with widely varying amplitude compared to oil- or water-filled reservoirs. Anomalous changes in seismic amplitude difference attributes for reflections within the reservoir are seen for gas/oil substitution. Oil/water substitution results in only weak variations of reservoir elastic properties, thus amplitude difference attributes may be unsuitable for monitoring changes in oil/water saturation. However, direct differences between the time traces are strong. On time-lapse sections, strong amplitude difference anomalies do not necessarily correspond to the region where the fluid variations occur; this is significant for monitoring oil field development by water injection.

\section{Introduction}

Reliable deduction of reservoir fluid properties from seismic data is vital in the quest for efficient hydrocarbon identification and extraction using primary, secondary and tertiary recovery techniques. Physical modelling allows theoretical numerical models to be tested, and physical models are particularly useful for quantitative studies of the variation of elastic properties arising from fluid substitution in reservoirs. They allow evaluation of the varying seismic response in realistic environments and situations; for example, in replicating field reflection surveys. We describe physical model experiments designed to develop and test fluid identification techniques, 
by fluid substitution in a layered and laterally heterogeneous reservoir model, and show that the analysis of the observations has important implications for the interpretation of time-lapse seismic data in hydrocarbon reservoirs, particularly for the location of the substituted fluids.

\section{Data Acquisition and Processing}

\subsection{Preparation of model}

The physical model aims to replicate a reservoir comprised of very thin layered sandstone and shale sequences, with lateral heterogeneity in terms of porosity, elastic parameters, etc. It consists of an overburden stratum and series of thin reservoirs with interbedded shale layers. The overburden layer is simulated with epoxy resin. Plexiglass plates represent thin shale layers, and artificial sandstone thin layers are made of $\mathrm{SiO} 2$ grains (150-300 meshes) mixed in epoxy resin, moulded at high temperature and pressure for good cementation. The interbedded model (see Figure 1a) has 7 sandstone layers and 8 shale layers.

\subsection{Parameter estimation}

Following the physical modeling experiments, the model was disassembled to measure the layer parameters. Microscope observations showed that the reservoir layers were laterally inhomogeneous, and provided data for porosity estimates. The model layer parameters are as follows:

(1) Overlying layer $50.2 \mathrm{~mm}$ thick, density $1.17 \mathrm{~g} / \mathrm{cm}^{3}$, P-wave velocity $2440 \mathrm{~m} / \mathrm{s}$.

(2) Each plexiglass plate thickness varies between $1.20 \mathrm{~mm}$ and $1.23 \mathrm{~mm}$.

(3) Porosity ranges from $9 \%$ to $24 \%$. The thickness of a single sand layer varies between $1 \mathrm{~mm}$ and $1.3 \mathrm{~mm}$ representing a $3 \mathrm{~m}$ to $5 \mathrm{~m}$ thick sand unit.

The seismic physical model is scaled to a realistic geological model, with the corresponding parameters listed in Table 1.

\subsection{Seismic physical modeling experiment}

The physical modeling experiment is performed in a water tank, and illustrated in Figure 1a. The water depth to the top of the model is $185 \mathrm{~mm}$. The wave source and receiver are located $0.5 \mathrm{~mm}$ 
under the water surface. The observation system is a single ended spread, with the source on the right and the hydrophone on the left, and this 2-D observation system is moved towards the left. The parameters of this system are given in Table 2. The source consists of an adjustable impulse generator, and a 23-bit analog-digital converter. The dominant frequency of the pulse is approximately $105 \mathrm{kHz}$. The seismic data are recorded in SEGY format.

Seismic experiments were conducted with the reservoir unit in the physical model filled with gas, water or oil, keeping all other parameters identical. Efforts were made to ensure the position of survey line to be the same in all experiments. Fluid substitution was performed very slowly (over 10 days) to simulate natural percolation and minimize disturbance to the well-cemented sand grains; no sand was found in the extracted fluids.

The seismic experiments were conducted as follows.

(1) With air filling the sand layers, the model is put into a vacuum thermotank and baked for 24 hours at $50^{\circ} \mathrm{C}$. The model sides are sealed to prevent water entry. The model is then sunk in the water tank and kept at a depth of $185 \mathrm{~mm}$. Seismic data are acquired, using the observation system listed in Table 2 .

(2) The seal is removed after experiment 1 . The interbedded layers are fully saturated with water through vacuum pumping, and this is repeated until the weight of the model does not increase anymore. All open sides are sealed again and the model is put into the water tank. Seismic measurements are carried out in the same way as for the first experiment.

(3) The seal is removed after experiment 2. The interbedded layers are put into a vacuum thermotank and the model is baked at $50^{\circ} \mathrm{C}$ and vacuum pumped every 2 hours. It is weighed 24 hours later to determine the amount of discharged water. This is repeated for 10 days, when the total amount of water discharged from the model in 10 days is $148 \mathrm{~g}$. In the last 3 days very little water was discharged. Then the model is saturated with engine oil using the same method as experiment 2 . The model is sealed again, put into the water tank, and the data acquisition is repeated.

Figure $1 \mathrm{~b}$ shows the raw data for three common shot gathers (shot numbers 17, 25, 34) from the third experiment. From the gathers, we note the reflection of water bottom/model interface is strong. The direct wave, random noise, and reflection of model bottom are also seen clearly. 


\subsection{Data Processing}

In order to analyze seismic reflection data acquired from the gas, water, and oil-filled physical models, we firstly upscale acquisition system according to Table 1. Band-pass filtering is applied to the raw data for noise suppression, and seismic sections of stacked profiles are obtained after velocity analysis. These sections are further processed by post stack migration (Francois, 1997). To avoid diffraction effects and side reflections at the model boundaries, only the traces between CDP 240-360 are used in reservoir characteristic analysis. Figures $2 \mathrm{a}, 2 \mathrm{~b}$ and $2 \mathrm{c}$ are the migration sections from the gas, water, and oil-filled models. Acquisition and processing parameters are the same for each experiment. From the migrated sections we observe:

(1) Horizontal events from the top of the overburden layer are very strong and the wave shapes are practically identical, as this layer does not change between experiments.

(2) The reflections from the water-filled and oil-filled reservoir show very similar wave shapes and amplitudes. The reservoir consists of overlapped horizontal layers, so the sand layer porosity, elastic modulus, and thus the reflection amplitude, vary laterally after the sand layer is filled with fluids.

(3) The reflections from the gas-filled and water-filled reservoir are clearly different. Seismic events from the gas filled layers show strong discontinuities, with large variations in energy level, and chaotic characteristics (wavy shapes).

The above phenomena demonstrate that (1) the reflection from a reservoir filled with gas is quite different from that from the same reservoir filled with liquid. There is no clear difference between the reflections from the water filled and oil filled reservoir, given the amplitude response for the respective sections; other seismic attributes are needed to resolve them. (2) For identical inhomogeneous horizontal layers, the events of gas filled model are discontinuous and the amplitudes are significantly different compared to the events from oil or water filled model. It is a reflection characteristic used to identify gas reservoir.

\section{Data Analysis and Interpretation}

To investigate differences in reflections from different fluid-filled reservoirs, time-lapse seismic 
techniques are applied to the data. The seismic data are processed using cross-equalization to keep non-reservoir reflections consistent, and thus focus on differences in the reservoir response (Li et al., 2001; Ross et al., 1996; Huang et al., 1998; Lumley, 2004). The observation from the oil filled reservoir is selected as the base survey, and the observations from gas- and water-filled reservoirs are used as monitoring surveys.

The cross-equalization technique needs a reference horizon in a non-reservoir area, so that its reflection is not affected by the variation of fluids. This is chosen to be the reflection at the interface between water and the top of the model, in the range between $750 \mathrm{~ms}$ and $850 \mathrm{~ms}$ in Figure 2. A matching filter can be designed from the reference horizon, then is applied to the monitoring data so as to maintain consistency between the non-reservoir reflections, while retaining the seismic differences due to fluid substitution in the reservoir $(860 \mathrm{~ms}-1000 \mathrm{~ms})$ (Huang et al., 1998).

This paper adopts six methods for differencing after cross-equalization (Bishop \& Nunns, 1994; Chen \& Sidney, 1997), as follows

(1) Direct subtraction method, in which seismic data difference is calculated at each time point between the base and monitoring traces.

(2) Root-mean-square (RMS) amplitude method.

(3) The difference between maximum amplitudes in the time window.

(4) The difference between minimum amplitudes in the time window.

(5) The difference between average amplitudes in the time window.

(6) The difference between amplitudes at the midpoint of the time window.

We applied time-lapse seismic attribute analysis firstly to the gas and oil model data. Figure 3a is the difference profile between the base and monitoring survey after cross-equalization. The difference for non-reservoir regions is very small, indicating the non-reservoir reflections are repeatable. However, the difference in the reservoir interbedded layer region (the 'reservoir layers') around $900 \mathrm{~ms}$ is large, and lateral variations are obvious. Thus in oil field exploitation with gas injection, the lateral variation of the reflection energy from the reservoir is noticeable and detectable. 
Figure 4a illustrates the differences in RMS amplitude between the gas- and oil-filled reservoirs. There is a positive anomaly in the reservoir layers, and a negative anomaly below the bottom of the reservoir layers. The maximum amplitude, minimum amplitude, average amplitude and midpoint amplitude difference attributes (not shown) display similar differences in characteristics between the reservoir layers and beneath the base of the reservoir. In all cases the difference attributes have flat features, but the lateral variations are large. This means that gas influences the reservoir attributes since the oil-filled horizontal reservoir shows only weak lateral differences. The maximum, minimum, and RMS amplitude differences show unique anomalies at the top and bottom of the reservoir, and it is easy to identify variations in the vertical direction. In general from the RMS, maximum, minimum amplitude difference profiles we observe that different fluid in the thin reservoir layers demonstrates quite different responses, i.e., the attribute anomalies are strong. The physical modeling experiments indicate that injected gas can be monitored in oil field development using gas injection.

Similar time-lapse analysis was applied to the data from the water- and oil-filled models. The migration profiles are shown in Figures $2 b, 2 c$ respectively. The profiles show that the characteristics of the reflections are similar, continuity is good, and the reflection from the top of the reservoir is clear. A matching filter was designed using these two data sets and applied to the water-filled model data. Figure $3 b$ shows the direct differences; it is observed that there are strong differences in reflection in the reservoir layers, even though the reflections appear similar (Figures $2 b, 2 c)$.

Figure $4 \mathrm{~b}$ shows the profile of RMS amplitude differences between the oil- and water-filled models. It is important to note that the anomalies corresponding to the reservoir are weak, whereas below the reservoir they are strong. Again, similar differences in characteristics between the reservoir layers and beneath the base of the reservoir are apparent for alternative difference attributes (not shown). Events below the reservoir exhibit clear positive differences for RMS and maximum amplitudes. The minimum amplitudes demonstrate a negative response, while those of average and midpoint amplitudes are alternatively positive and negative.

Examining these profiles (Figure $4 \mathrm{~b}$ is an example) and Figure $3 \mathrm{~b}$, it is clear that the various attributes show different behaviour. Direct differences between cross-equalized profiles are larger 
in the reservoir than above or below it. In contrast, differences of average, maximum, minimum, midpoint and RMS amplitudes corresponding to the reservoir are small, while those below the reservoir are large.

These characteristics are important for the understanding and interpretation of time-lapse seismic difference profiles. When the fluid filling a reservoir changes, the reservoir elastic properties change. The changes cause differences in travel times, amplitudes, spectra and phases of the reflections related to the reservoir and underlying strata. If the inhomogeneity of the reservoir is strong, cross-equalization cannot fully eliminate the travel time differences. Direct subtraction of two profiles gives rise to some errors, due to the travel time differences, so the anomalies corresponding to the reservoir are large.

In the profiles of average, maximum, minimum, midpoint, RMS amplitude difference attributes, anomalies corresponding to the reservoir are small. This is because the subtraction is conducted after trace alignment. In Figure $4 \mathrm{~b}$, the small amplitude anomalies corresponding to the reservoir indicate that oil/water substitution results in relatively weak changes in elastic parameters. These five attributes have low accuracy in detecting oil/water substitution in timelapse seismic exploration.

Figure $4 \mathrm{~b}$ shows that the differences below the reservoir are much larger than those in the reservoir. From the point of view of travel time the reflections are related to the interbed multiples within the thin layers. These multiples cannot be suppressed completely in data processing since the layers are too thin. In addition, the reservoir lateral inhomogeneity further contributes to the presence of multiples inside the layers. Small variations in elastic differences due to fluid substitution cause large differences in these multiples, amplifying the attribute difference. As a result, the amplitude anomalies shown in Figure $4 \mathrm{~b}$ are due to multiples. Thus differences in attributes may not represent the equivalent magnitude of fluid variation, especially when observed in the region below the reservoir. This phenomenon may occur in the development of inhomogeneous reservoir by water injection.

\section{Conclusions}

A simulation of a time-lapse seismic experiment was conducted on a physical model of a 
hydrocarbon reservoir with inhomogeneous thin interbedded sand and shale layers, using different fluids. Analysis of the data produced the following results:

(1) Reflection data from a gas-filled reservoir is quite different to reflection data from a water or oil-filled reservoir. For inhomogeneous horizontal layers, reflections in a gas-filled model are discontinuous and their amplitudes vary dramatically compared to an oil- or water-filled model. This reflection characteristic could be used to distinguish a gas reservoir.

(2) Substitution of gas for oil, or vice versa, in a thin interbedded reservoir results in significant anomalies in the seismic attribute difference profiles. This indicates that time-lapse seismics are able to monitor fluid changes during an enhanced oil recovery process by steam or gas injection.

(3) Reflection from the oil-filled reservoir is quite similar to the one from the water-filled reservoir. Oil/water substitution causes only weak variations of the elastic properties of reservoir, but direct differences between the time traces are strong. Time-lapse seismic attribute differences involving average amplitude, maximum amplitude, minimum amplitude, midpoint amplitude, RMS amplitude, have weak anomalies in the reservoir, thus are likely to be difficult to use for monitoring changes in reservoir oil/water saturation.

(4) On the profiles of the 5 attributes, interbed multiples show large differences, which appear to originate below the reservoir. This illustrates that on time-lapse seismic sections, the strong anomaly region does not necessarily correspond to the region where the fluid variations occur. This may commonly occur in non-uniform oil field development by water injection.

(5) If the reservoir is strongly laterally inhomogeneous, cross-equalization cannot completely eliminate the time difference between two seismic observations, thus direct subtraction of the poststack migration profiles may generate mixed differences which can be large. However, the large anomalies do not represent strong impedance differences caused by oil/water substitution.

\section{Acknowledgments}

This research is supported by "973" project (2007CB209600) of the Ministry of Science and Technology of China, and State Key Lab of Gas and Oil Resources and Exploration. Dr. Lishan 
Cui made the physical model, and Dr. Xuri Huang guided the time-lapse seismic data processing. We thank Fred Hilterman and an anonymous reviewer for constructive comments and input. This paper is published with the permission of the Executive Director of the British Geological Survey (NERC).

\section{References}

Bishop, T. N., and A. G. Nunns (1994), Correcting amplitude, time, and phase mis-ties in seismic data, Geophysics, 59, 946-953.

Chen, Q., and S. Sidney (1997), Seismic attribute technology for reservoir forecasting and monitoring, The Leading Edge, 16, 445-456.

Francois, A., D. Nichols, T. Rekdal, B. Biondi, D. E. Lumley, and H. Urdaneta (1997), Imaging complex geologic structure with single-arrival Kirchhoff prestack depth migration, Geophysics, $62,1533-1543$.

Huang, X., L. Meister, and R. Workman (1998), Improving production history matching using time-lapse seismic data, The Leading Edge, 17, 1430-1433.

Li, G. P., G. Purdue, S. Weber, and R. Couzens (2001), Effective processing of nonrepeatable 4-D seismic data to monitor heavy oil SAGD steam flood at East Senlac, Saskatchewan, Canada, The Leading Edge, 20, 54-62.

Lumley, D. E. (2004), Business and technology challenges for 4D seismic reservoir monitoring: The Leading Edge, 23, 1166-1168.

Ross, C. P., G. B. Cunningham, and D. P. Weber (1996) Inside the cross-equalization black box, The Leading Edge, 15, 1233-1240.

\section{Figure captions}

Figure 1. (a) Physical model and seismic observation system. The model is in water, the overburden layer and interbedded layers show $\mathrm{x} 2$ enlargement in the vertical direction. The 
observation system is $0-20-336 \mathrm{~mm}$ (corresponding to $0-60-1008 \mathrm{~m}$ in field scale ). 50 shots were made with 80 traces for each shot. The intervals of shot and receiver are $10 \mathrm{~mm}$ (corresponding to $30 \mathrm{~m}$ ) and $4 \mathrm{~mm}$ (corresponding to $12 \mathrm{~m}$ ). (b) Three raw common shot gathers (shot 17, 25, 34) from the oil-filled model; numbered wave arrivals are (1) direct wave, (2) the reflection of water bottom/model interface, (3) random noise, (4) reflection at the reservoir, (5) reflection at the bottom of the model.

Figure 2. Partial migration sections for models filled with (a) gas, (b) water, and (c) oil. "Water bottom" indicates the reflection of the interface between water and model. "Thin interbed" indicates reflections of many interfaces between thin sand and shale layers.

Figure 3. The direct difference profiles (a) between the base (oil-filled) and monitoring (gas-filled) surveys, and (b) between the water-filled measurement and oil-filled measurement, after crossequalization.

Figure 4. RMS P-wave amplitude differences for amplitudes recorded in (a) gas- and oil-filled reservoirs, and (b) water- and oil-filled reservoirs. 
Table 1. The relation between the physical model and a real geological model.

\begin{tabular}{llll}
\hline Parameter & Geological stratum & Physical model & Scale \\
P wave velocity $\left(V_{p}\right)$ & $2500 \mathrm{~m} / \mathrm{s}$ & $2500 \mathrm{~m} / \mathrm{s}$ & $1: 1$ \\
Dominant frequency $(f)$ & $35 \mathrm{~Hz}$ & $105 \mathrm{kHz}$ & $1: 3000$ \\
Dominant wavelength $(\lambda)$ & $71 \mathrm{~m}$ & $24 \mathrm{~mm}$ & $3000: 1$ \\
Thickness of thin layer $(h)$ & $<5 \mathrm{~m}$ & $1-1.7 \mathrm{~mm}$ & $3000: 1$ \\
Temporal sampling interval & $0.6 \mathrm{~ms}$ & $0.2 \mu \mathrm{s}$ & $3000: 1$ \\
Model dimensions & $13500 \mathrm{~m} \times 900 \mathrm{~m}$ & $450 \mathrm{~mm} \times 300 \mathrm{~mm}$ & $3000: 1$ \\
Depth to top of target layer & $150 \mathrm{~m}$ & $50.2 \mathrm{~mm}$ & $3000: 1$ \\
Depth of water layer & $556 \mathrm{~m}$ & $185 \mathrm{~mm}$ & $3000: 1$
\end{tabular}


Table 2. Parameters of seismic observation system.

\begin{tabular}{lll}
\hline & Physical model & Geological model \\
Observation system & $0-20-316 \mathrm{~mm}$ & $0-60-1008 \mathrm{~m}$ \\
Trace interval & $4 \mathrm{~mm}$ & $12 \mathrm{~m}$ \\
Shot interval & $10 \mathrm{~mm}$ & $30 \mathrm{~m}$ \\
Smallest offset & $20 \mathrm{~mm}$ & $60 \mathrm{~m}$ \\
Largest offset & $336 \mathrm{~mm}$ & $1008 \mathrm{~m}$ \\
Number of traces & 80 & 80 \\
Number of shots & 50 & 50 \\
\hline
\end{tabular}




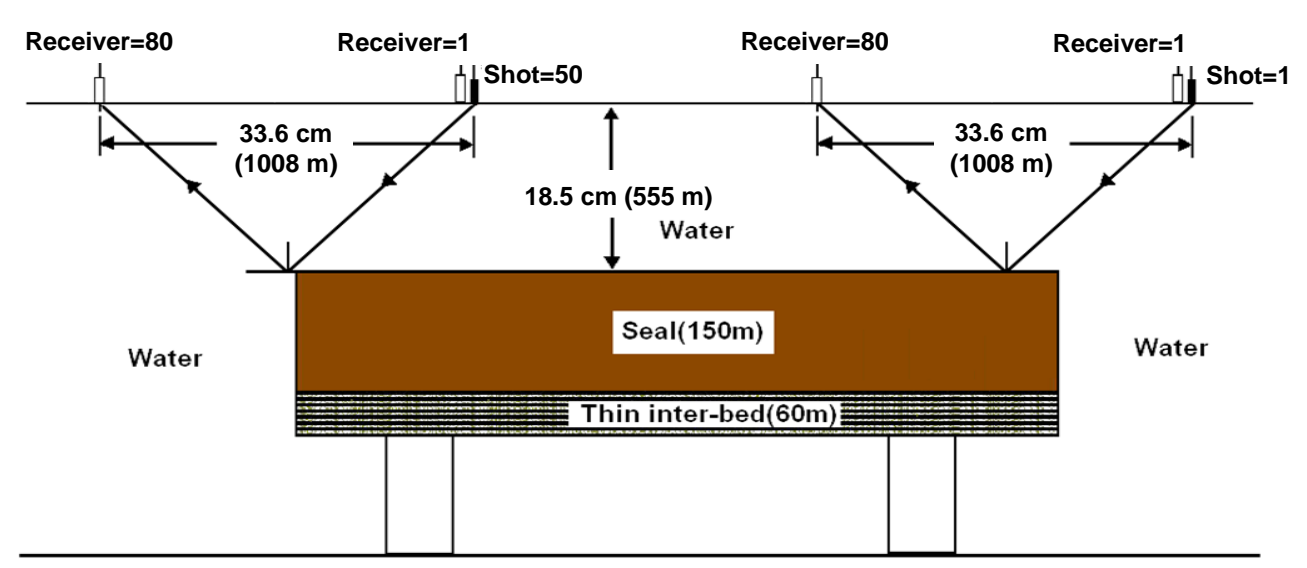

(a)

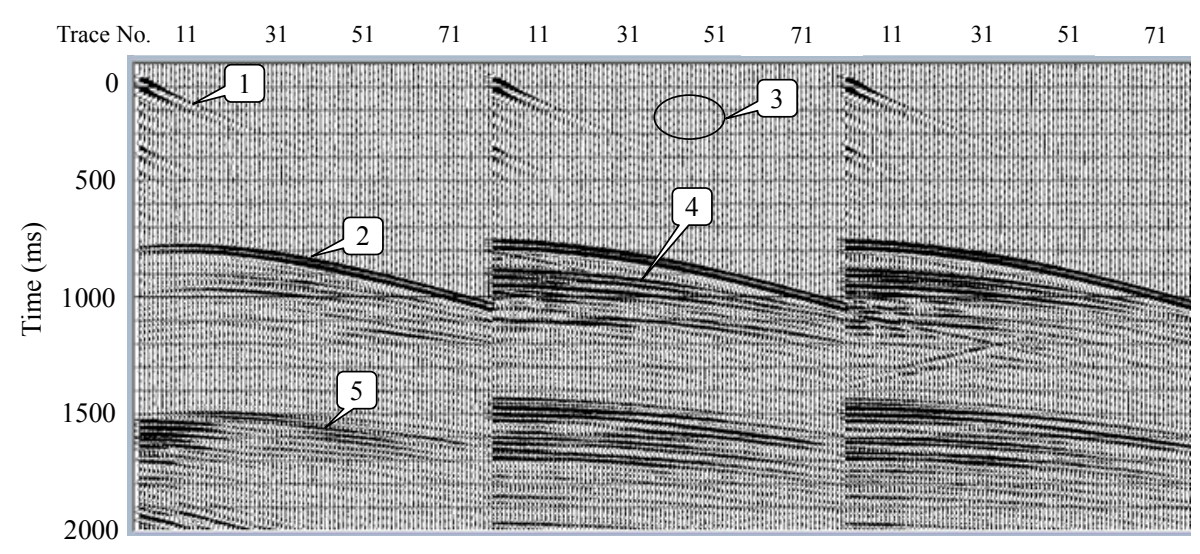

(b)

Figure 1.

Figure 1. (a) Physical model and seismic observation system. The model is in water, the overburden layer and interbedded layers show $\mathrm{x} 2$ enlargement in the vertical direction. The observation system is $0-20-336 \mathrm{~mm}$ (corresponding to $0-60-1008 \mathrm{~m}$ in field scale ). 50 shots were made with 80 traces for each shot. The intervals of shot and receiver are $10 \mathrm{~mm}$ (corresponding to $30 \mathrm{~m}$ ) and $4 \mathrm{~mm}$ (corresponding to $12 \mathrm{~m}$ ). (b) Three raw common shot gathers (shot 17, 25, 34) from the oil-filled model; numbered wave arrivals are (1) direct wave, (2) the reflection of water bottom/model interface, (3) random noise, (4) reflection at the reservoir, (5) reflection at the bottom of the model. 


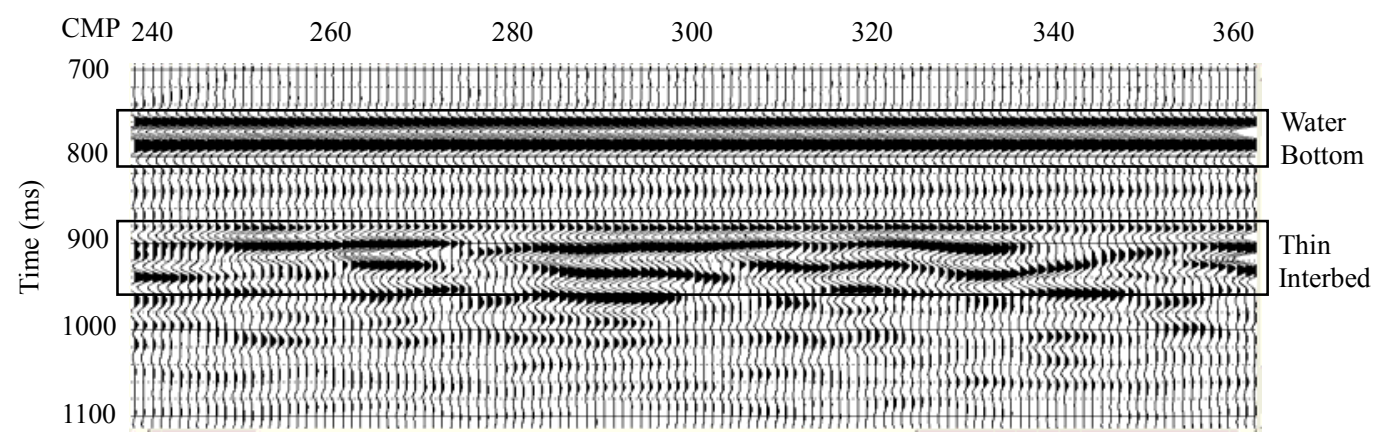

(a)

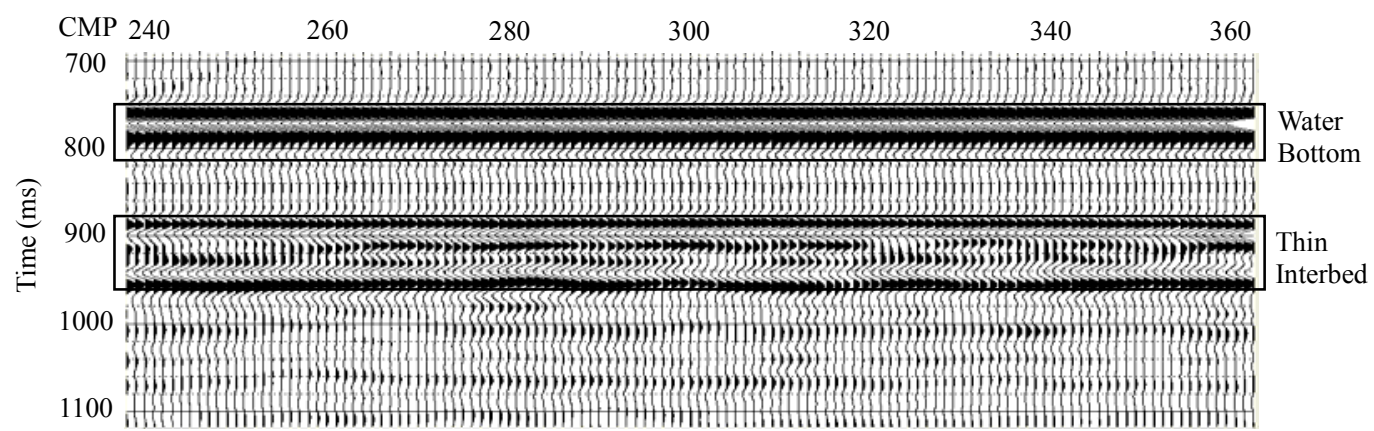

(b)

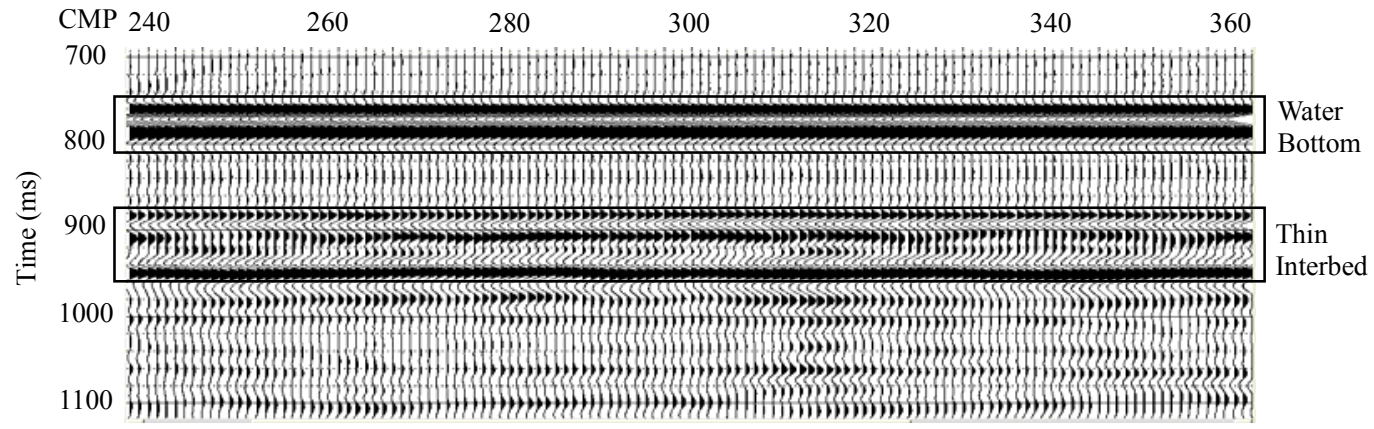

(c)

Figure 2. Partial migration sections for models filled with (a) gas, (b) water, and (c) oil. "Water bottom" indicates the reflection of the interface between water and model. "Thin interbed" indicates reflections of many interfaces between thin sand and shale layers. 


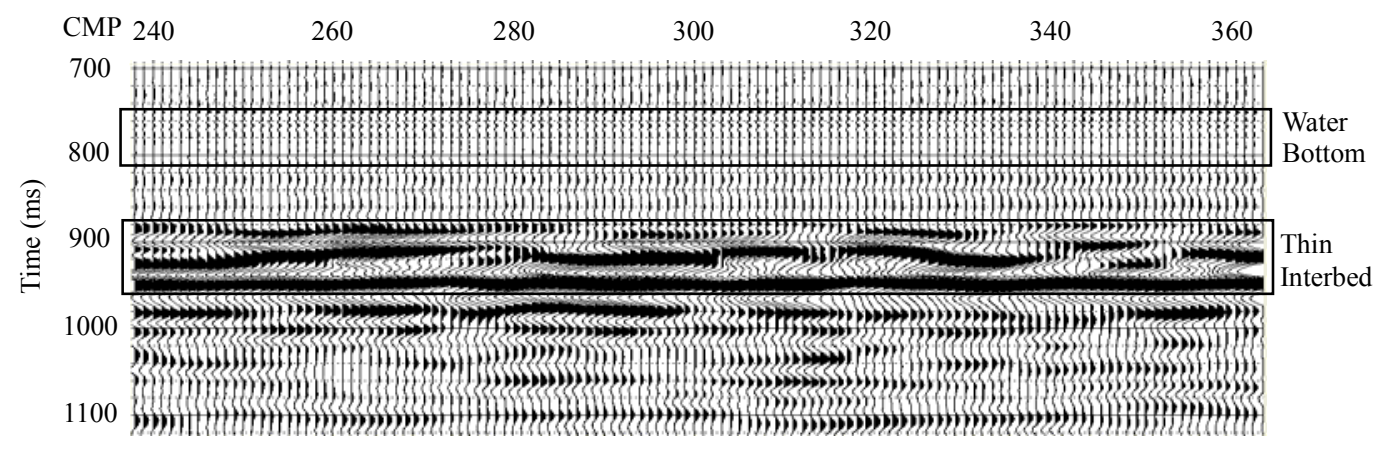

(a)

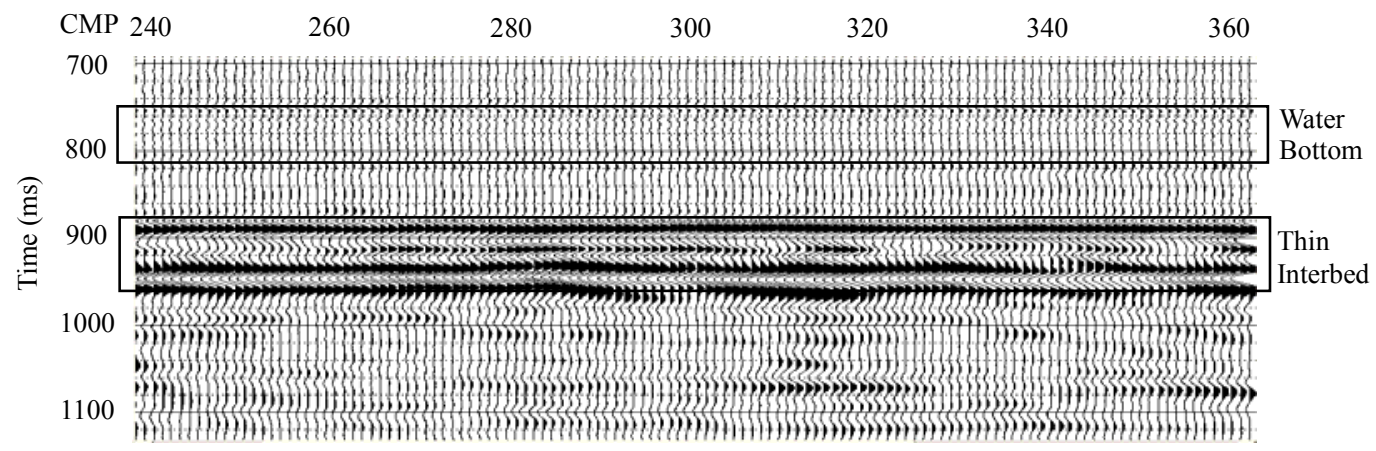

(b)

Figure 3. The direct difference profiles (a) between the base (oil-filled) and monitoring (gas-filled) surveys, and (b) between the water-filled measurement and oil-filled measurement, after crossequalization. 


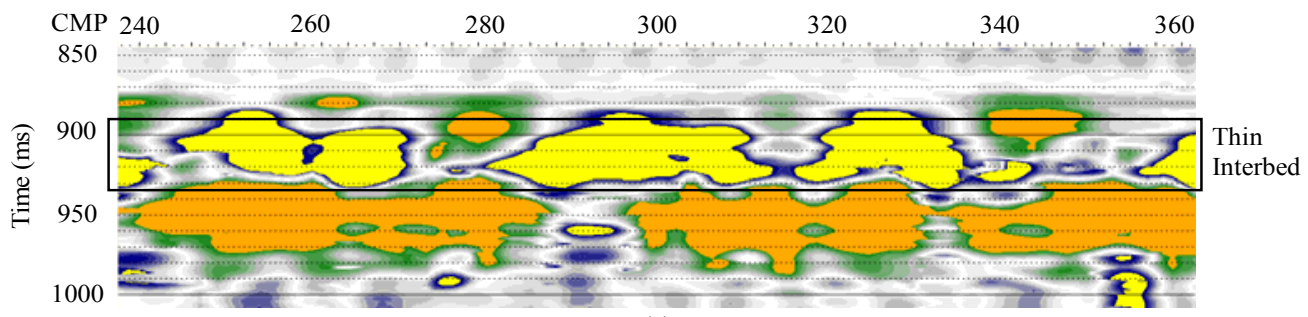

(a)
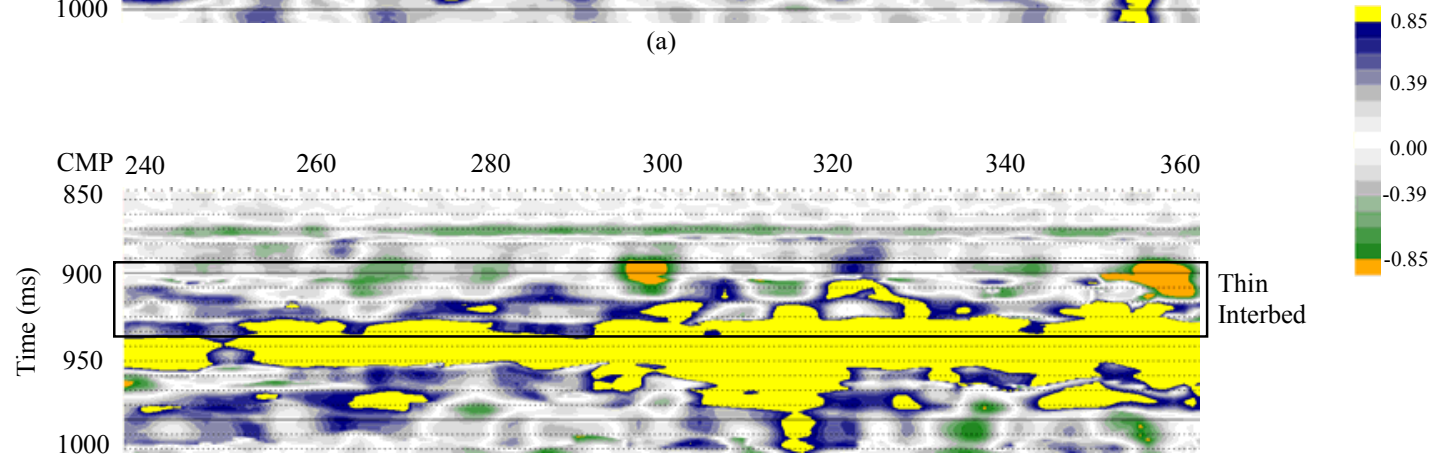

(b)

Figure 4. RMS P-wave amplitude differences for amplitudes recorded in (a) gas- and oil-filled reservoirs, and (b) water- and oil-filled reservoirs. 
